\title{
Mixed-list manipulations of implicit associative responses in verbal discrimination learning
}

\author{
N. JACK KANAK* and KAREN N. JONES \\ University of Oklahoma, Norman, Oklahoma 73069
}

\begin{abstract}
Implicit associative responses were manipulated in a verbal discrimination task involving mixed-list of (a) pairs containing associatively related wrong (W) and right (R) items (intrapair condition), (b) associatively related $\mathrm{W}$ and $\mathrm{R}$ items in different pairs (interpair condition), (c) associatively related $\mathrm{R}$ items (interitem-AR condition), and (d) pairs involving no associative relationships (control condition). In agreement with frequency theory, the interitem-AR condition resulted in pronounced facilitation in acquisition relative to the control and interpair conditions, a result not consistently found with unmixed lists. Consistent with the "tagging" model but contrary to frequency theory, the intrapair condition was also significantly superior to the control and interpair conditions. The control and interpair conditions did not differ, suggesting that the interference usually found in the interpair condition in unmixed lists may be largely inhibited under the present mixed-list conditions.
\end{abstract}

The frequency theory (Ekstrand, Wallace, \& Underwood, 1966) of verbal discrimination (VD) learning predicts that the presence of associatively related items in a list may facilitate or inhibit list acquisition, according to whether the implicit associative response (IARs, Bousfield, Whitmarsh, \& Danick, 1958) acts to increase or reduce the intrapair differential subjective frequency between the right (R) and wrong (W) items. According to the theory, lists containing associatively related $\mathrm{W}$ and $\mathrm{R}$ items in two different pairs (interpair condition) or within a single pair (intrapair condition) should be more difficult to learn than a control list of unrelated items, since the differential frequency cue is reduced by the increment in subjective frequency of the $\mathrm{W}$ items caused by the IAR to $\mathrm{R}$ items. However, when the list contains associatively related $R$ items and unrelated $\mathrm{W}$ items (interitem-AR), learning should be facilitated relative to the control condition.

The predicted increased difficulty for interpair manipulations has received substantial support (e.g., Eberlein \& Raskin, 1968; Fulkerson \& Kausler, 1969). Support for the same prediction for the intrapair condition has been more elusive. Palermo and Ullrich (1968) observed the effect consistently over four experiments, and McCarthy (1973) essentially replicated these results by demonstrating that high assiciate pairs were more difficult to learn than low and zero associate pairs. Others (e.g., Eberlein \& Raskin, 1968; Fulkerson \& Kausler, 1969; Lovelace \& Schulz, 1971), however, have reported null differences for the intrapair vs control comparison. Fulkerson and Kausler attributed the null difference to a compensatory mechanism of "tagging and collapsing." That is, after $\mathrm{S}$ recognizes the presence of an intrapair association, during the feedback interval, it is suggested that he uses the strategy of "tagging" the underlined $\mathrm{R}$ item as "correct" and then collapses the tag over the association for memory storage (e.g., rehearses "table-chair," "chair is correct"). On the

*Requests for reprints should be addressed to N. Jack Kanak, Department of Psychology, University of Oklahoma, Norman, Oklahoma 73069 . subsequent anticipation trial $S$ need only recognize the intrapair association (table-chair) upon presentation of the pair and then retrieve the "tag" (chair).

Two potentially important methodological differences were involved, however, between those intrapair studies which support the frequency theory and those which do not. Both Palermo and Ullrich (1968) and McCarthy (1973) used a 1:1-sec rate of presentation and mixed lists with pairs of varying associative strength, while the studies not supporting the theory used a $2: 2-\mathrm{sec}$ rate and unmixed lists. It is conceivable that a $1: 1-\mathrm{sec}$ rate does not allow adequate time for the utilization of a compensatory "tagging" process, while the use of a mixed list of pairs of varying associative strength, or the contrast of item function of the associates, or both, might draw S's attention to the high associative strength pairs, thus activating the IAR and resulting in a reduction of the differential frequency cue without adequate time for the compensatory tagging process to be involved. As Lovelace and Schulz (1971) have pointed out, a mixed-list manipulation of the presence or absence of associative relationships may introduce selective and differential attention to the attributes of the materials. Although the present study was not designed to determine whether these methodological differences do account for the discrepant results regarding the intrapair condition, the logic above regarding the effect of using mixed lists was intentionally applied.

Although Ekstrand et al (1966) reported significant facilitation for the interitem-AR condition, consistent with the frequency theory, a series of six experiments (Kanak, Cole, \& Thornton, 1970; Kanak, Cole, \& Eckert, 1972) reproduced this finding in only one experiment, and only then when Ss were instructed regarding the associative relationship, despite the manipulation of a number of potentially pertinent methodological variables. Similarly, the dominance or associative strength of the relationship between related $\mathrm{R}$ items failed to be defined as a significant variable (Mueller, Kanak, \& Flannagan, in press) in an 
interitem-AR manipulation, in contrast to a successful similar manipulation (though within a mixed list) in the intrapair condition (McCarthy, 1973).

The operation of the IAR mechanism per se in interpair and intrapair manipulations, though in possibly a different manner from that predicted by the theory in some studies dealing with the latter condition, seems well established. It is therefore puzzling that its operation has not been more consistently demonstrated in the interitem-AR condition. Kanak et al (1972) pointed out that the interpair and intrapair manipulations involve a contrast of function (i.e., W or $\mathrm{R}$ ) of the associatively related items which may serve as a sufficient cue to activate attention to the associative relationship even in unmixed lists. The fact that Eberlein and Raskin (1968) found comparable interference for the interpair condition without and with instructions regarding the list composition is consistent with this notion. In an unmixed-list interitem-AR condition, however, an item function contrast cue is not present to enhance S's awareness of the associative relationships present. Furthermore, the absolute effect of IARs may often be relatively subtle since the normative values for frequency of the primary associate rarely exceed $60 \%$, and equality of bidirectional strength of the association is not frequent.

The present study used a mixed-list procedure to attempt to deliberately introduce selective attention to the attributes of the materials in order to produce an optimal situation for the activation of the IAR process in the interitem-AR condition. The mixed list employed pairs representing not only this condition, but also the intrapair, interpair, and control conditions. If S's awareness of IARs or the presence of associates is mediated by the contrast of item function of the associates in the intrapair and interpair condition, or by lack of their presence in control pairs, such awareness should generalize to the interitem-AR condition and produce facilitation relative to control pairs as a result of the increment in subjective frequency of the $R$ items produced by the IAR. In addition, if the compensatory tagging process is operative in the intrapair condition, these pairs should produce significantly fewer errors than control pairs but significantly more if the prediction from frequency theory is supported. Although previous literature with unmixed lists has shown that the interpair condition produces the interference predicted by frequency theory, this prediction has not been tested in a mixed list.

\section{METHOD}

\section{Subjects}

Forty-eight students from introductory psychology classes at the University of Oklahoma served as Ss in partial fulfillment of a course requirement. All Ss were naive with respect to participation in prior verbal learning experiments.

\section{Lists}

Each list contained 20 pairs of words: 4 intrapair, 4 interpair, 8 interitem-AR, and 4 control. Eight interitem-AR pairs were employed in order to represent four associative relationships, as in the other two experimental conditions. The associatively related words were chosen from Palermo and Jenkins (1964) norms and had a mean associative frequency of $47 \%$, and all words used had an A or AA frequency. The control words also had A or AA frequency and were associatively unrelated. Six lists were formed using each set of four associa tively related pairs of words in each of the three types of IAR relationships, and a second form of each list was used in which the $\mathrm{R}$ items in the intrapair, interpair, and control pairs of one form served as $W$ items in the other form to control for possible differential difficulty related to word function. Four random serial orders were used for each of the 12 lists with the restrictions that no more than three $\mathbf{R}$ items appeared in succession in a given spatial position and that two pairs of the same treatment type never appeared in succession. In each list, each word unsystematically occurred twice on the left or right across the four random serial orders as a control for spatial-position effects.

\section{Procedure}

The Ss were assigned to the various lists depending upon their order of appearance at the laboratory. All Ss were tested individually and given standard instructions for a VD learning task. A Lafayette memory drum was used to present the lists by the anticipation method with a $2: 2 \mathrm{sec}$ presentation rate and a 4-sec intertrial interval. During the anticipation interval, each pair appeared in horizontal alignment and the $S$ made his response by pronouncing the word aloud. The same pair appeared again in the feedback interval with the correct word underlined. All Ss learned the list to a criterion of two successive perfect trials. The data were not used for those Ss who did not attain criterion within 20 trials and the three Ss who failed to meet this criterion were replaced by the next Ss on the schedule.

\section{RESULTS AND DISCUSSION}

The data for errors was reduced to the proportion of errors to the base of opportunities to provide a common index, because of the differential number of pairs to be learned among the treatment conditions. The total number of errors to the criterion of two successive perfect trials was tabulated for each $S$ for those pairs within each treatment condition, and this value was divided by the product of the number of items in that condition multiplied by the number of trials $S$ required to achieve the criterion for all pairs in that condition. A one-way analysis of variance with repeated measures indicated a significant effect for treatment conditions, $F(3,141)=6.00, p<.01$. The mean proportions and standard deviations, respectively, were $.209, .092$ (intrapair), .212, .060 (interitem-AR), .248, .075 (interpair), and .260, .084 (control). Individual comparisons using the residual error term $(.0055)$ and Tukey's HSD test indicated that the error rate was significantly $(p<.01)$ less for both the intrapair and interitem-AR conditions relative to the control. The difference between the interpair and control conditions was clearly nonsignificant. In addition, both the intrapair and interitem-AR conditions produced a significantly $(\mathrm{p}<.05)$ lower error rate than the interpair condition.

The interitem-AR and intrapair conditions were significantly superior to the control condition, as well as to the interpair condition. The interitem-AR condition therefore supports the frequency theory while the 
intrapair condition does not, but rather supports the postulated compensatory tagging mechanism (Fulkerson \& Kausler, 1969). Although those studies earlier cited as reporting null differences between the intrapair and control conditions may be viewed as implying support for a tagging mechanism, the significant difference in the present study represents more substantial evidence favoring such a mechanism. The stronger effect in this study and the superiority of the interitem-AR condition to the control condition, a result which heretofore has been most elusive, may both be due to the selective attention to list attributes which a mixed-list design may encourage (Lovelace \& Schulz, 1971). That is, S's exposure to three different types of associative relationships may have allowed him to recognize much earlier the opportunity to utilize a tagging mechanism in intrapair associate pairs than occurs in unmixed-list designs. The superiority of the interitem-AR condition suggests that the IAR mechanism was indeed activated in pairs of that type and that, as a result, the differential subjective frequency cue became more discriminable earlier than for control pairs.

The failure to find the interpair condition inferior to the control condition does not support the frequency theory or the findings of others previously cited who employed unmixed lists. However, it is possible that $S$ could utilize his awareness of the positive contribution of associates in the intrapair and interitem-AR conditions to minimize the expected inhibitory influence, once experienced, of associates in the interpair condition. Indeed, in the present mixed-list design, if $S$ is capable of utilizing a process of a higher level than simple differential frequency unit accrual as our interpretation of the results of the intrapair condition suggests, it is plausible that in the context of a mixed list of treatments he might attempt to apply higher-order strategies to all types of pairs involving associates. Such multiple strategies might involve S's attending to the associates if they involve $W$ and $R$ items in the same pair, using a "tagging" strategy; attending to them if the associates involve only $\mathrm{R}$ items, using an "approach" strategy (Cole \& Kanak, 1972); and ignoring associations and relying on rote memory if they involve $\mathrm{W}$ and $\mathrm{R}$ items in different pairs, a strategy similar to the "avoidance" strategy Cole and Kanak suggested was involved in their "instructed" interitem-AW condition. A second possible strategy in the interpair condition is to use a "tagging" approach with S labeling "chair is correct," "table is incorrect" and recalling the appropriate tag to mediate his response when either of the pairs is presented involving a given interpair association. A test of whether such multiple strategies are employed might be conducted by varying rate of presentation within a mixed-list design. For example, a $1: 1-\mathrm{sec}$ rate, as opposed to the $2: 2 \mathrm{-sec}$ rate used in the present study, should not allow time for the utilization of such strategies if our hypotheses are correct concerning the reasons for the differences in the results of Palermo and Ullrich (1968) and McCarthy (1973) vs Eberlein and Raskin (1968), Fulkerson and Kausler (1969), and Lovelace and Schulz (1971). On the other hand, if the mixed-list design induces selective attention to list attributes, and if the $1: 1-\mathrm{sec}$ rate of presentation does not allow time for the use of strategies, IAR processes should still be activated and the predictions of frequency theory should be supported in all three conditions.

Although this discussion of the use of multiple strategies may only be appropriate to similar mixed-list designs with similar procedures, and is admittedly ad hoc with respect to the interpair condition, it suggests that mixed-list designs may have utility for studying strategies, even while recognizing that such strategies or processes may not always be generalizable to comparable manipulations in unmixed-list designs. Nevertheless, practical discrimination problems often involve "mixed-list" characteristics and certainly occur "within-Ss." In addition, the use of a mixed-list, within-S design in the investigation of IAR processes, where previous research with unmixed lists has often left an ambiguous picture, has the advantage of holding constant within Ss and, at the same time, across treatment conditions the previously cited shortcoming that associative strength of normative primary associates, based on between-S norms, rarely exceeds $60 \%$ and is frequently not of equal bidirectional strength.

\section{REFERENCES}

Bousfield, W. A., Whitmarsh, G. A., \& Danick, J. J. Partial response identities in verbal generalization. Psychological Reports, 1958, 4, 703-713.

Cole, E. E., \& Kanak, N. J. Transfer of implicit associative responses between free-recall learning and verbal discrimination learning tasks. Journal of Experimental Psychology, 1972, 95, 110-115.

Eberlein, E., \& Raskin, D. C. Intrapair and interpair associations in verbal discrimination learning. Psychonomic Science, 1968, $11,145-146$.

Ekstrand, B. R., Wallace, W. P., \& Underwood, B. J. A frequency theory of verbal discrimination learning. Psychological Review, 1966, 73, 566-578.

Fulkerson, F. E., \& Kausler, D. H. Supplementary report: Effects of intrapair and interpair bidirectional associates on verbal discrimination learning. Journal of Verbal Learning \& Verbal Behavior, 1969, 8, 307-310.

Kanak, N. J., Cole, L. E., \& Eckert, E. Implicit associative responses in verbal discrimination acquisition. Journal of Experimental Psychology, 1972, 93, 309-319.

Kanak, N. J., Cole, L. E., \& Thornton, J. W. Interitem manipulations of implicit associative responses in verbal discrimination learning. Psychonomic Science, 1970, 18, 359-361.

Lovelace, E. A., \& Schulz, L. S. Intrapair associations in verbal discrimination learning. Psychonomic Science, 1971, 24, 157-158.

McCarthy, S. V. Verbal discrimination learning as a function of associative strength between noun pair members. Journal of Experimental Psychology, 1973, 97, 270-271.

Mueller, J. H., Kanak, N. J., Flanagan, J. L. Implicit associative-response strength in verbal discrimination. American Journal of Psychology, in press.

Palermo, D. S., \& Jenkins, J. J. Word association norms: Grade school through college. Minneapolis: University of Minnesota Press, 1964.

Palermo, D. S., \& Ullich, J. R. Verbal discrimination learning as a function of associative strength between word-pair members. Journal of Verbal Learning \& Verbal Behavior, 1968, 7, 945-952.

(Received for publication December 17, 1973.) 